\title{
Resistencia a la fractura frente a carga estática transversal en piezas dentarias restauradas con espigo-muñón colado, postes de fibra de carbono y de aleación de titanio
}

Quintana M, Castilla M, Matta C. Resistencia a la fractura frente a carga estática transversal en piezas dentarias restauradas con espigo-muñón colado, postes de fibra de carbono y de aleación de titanio. Rev Estomatol Herediana 2005;15(1): 24 - 29

\section{RESUMEN}

El propósito del presente estudio fue determinar la resistencia a la fractura frente a carga estática transversal de piezas dentarias tratadas endodonticamente, restauradas con espigomuñón colado, postes de fibra de carbono(C-Post ${ }^{\circledR}$, (Bisco Inc) y postes de aleación de titanio (Parapost ${ }^{\circledR}$, (C) Coltène/Whaledent Inc.). Se utilizó el adhesivo All Bond $2^{\circledR}$ (CBisco, Inc) como material adhesivo y el cemento de resina Duolink ${ }^{\circledR}$ (CBisco Inc) para cementar los postes. La prueba de anova unifactorial encontró que las piezas dentarias restauradas con espigo-muñón colado tuvieron una mayor resistencia frente a carga estática transversal que las piezas dentarias restauradas con postes de fibra de carbono(C-Post ${ }^{\circledR}$, CBisco Inc) y postes de aleación de titanio (Parapost ${ }^{\circledR}$, @ Coltène/Whaledent Inc).

\section{Palabras clave: TÉCNICA DE PERNO MUÑON / FUERZA COMPRESIVA}

Fracture resistance to static transversal loading on teeth restored with post-core, fibre post carbon and post titanium alloy

ABSTRACT

The purpose of this study was to determine fracture resistance to static transversal loading of endodontically treated teeth restored with post-core, fibre post carbon (C-Post ${ }^{\circledR}$, ( $\mathrm{CBisco}$ Inc) and post titanium alloy (Parapost ${ }^{\circledR}$, (C)Coltène/Whaledent Inc). All Bond $2{ }^{\circledR}$ (CBisco, Inc) was used as a bonding material and Duolink ${ }^{\circledR}$ (@Bisco Inc) resin cement for luting the post. The differences among the scores were tested for statistical significance $(\mathrm{p}<0.05)$ and results show that treated teeth restored with post-core had a major fracture resistance to static transversal loading than those restored with fibre post carbon (C-Post ${ }^{\circledR}$, (CBisco Inc) and post titanium alloy (Parapost ${ }^{\circledR}$, (C) Coltène/Whaledent Inc).

Keywords: POST AND CORE TECHNIQUE / COMPRESSIVE STRENGTH

\section{Introducción}

Actualmente se sabe que el poste no sirve para reforzar el diente tratado endodónticamente, este provee de retención para coronas y pilares de puente (1-3). Los postes no fortalecen los dientes y la perdida de estructura dental por la preparación, debilitan la raíz, incrementando la probabilidad de fractura radicular (4).

El poste debe tener una longitud por lo menos equivalente a los dos tercios de la longitud radicular. El diámetro del poste a medida que aumenta mejora la retención del mismo, a causa de una mayor superficie disponible. Como promedio es conveniente que el orificio para el poste sea de unos $2 \mathrm{~mm}$ de diámetro. Se puede mejorar la retención a expensas del diámetro del diente pero el riesgo de fractura aumenta notablemente (2,5-7).

El espigo-muñón colado tiene la ventaja de su conformación íntima a la configuración del conducto radicular preparado. Su desventaja es la demanda de mayor tiempo en su elaboración, así como un difícil retiro del conducto si fuera necesario, posibilidad de corrosión y necesidad de desgastar parte de la estructura coronal $(2,8,9)$.

Las ventajas de los postes prefabricados son su fácil uso, posibilidad de ser colocados en una sola sesión terapéutica, pueden ser retirados con mayor facilidad, no se corroen y ahorro de tiempo. Su desventaja es su difícil aplicación cuanto mayor es la pérdida dental (10-12).

Los postes prefabricados de acuerdo al material empleado para su fabricación pueden ser de acero inoxidable, aleación de titanio, fibra de carbono, fibra de vidrio y zirconio $(6,13,15)$.

Los postes prefabricados metálicos

\section{Martín Quintana del Solar Marisol Castilla Camacho Carlos Matta Morales}

Docente del Departamento Académico de Clínica Estomatológica. Facultad de Estomatología. Universidad Peruana Cayetano Heredia.

\section{Correspondencia}

Martín Quintana del Solar, Los Jazmines 523, Lima 14 - Perú. E-mail:martin34@ec-red.com

Aceptado para publicación : 22 de junio del 2005 cilíndricos de lados paralelos, distribuyen mejor las tensiones dentro de la raíz en comparación a los de forma cónica $(1,13,14)$. Los postes prefabricados de aleación de titanio, poseen un menor módulo de elasticidad con respecto a los confeccionados de acero inoxidable, generando una menor tensión dentro del conducto radicular, por consiguiente una menor fractura radicular $(16,17)$.

El poste prefabricado de fibra de carbono C-Post ${ }^{\circledR}$ de la empresa (CBisco Inc, está fabricado de fibras de carbono estiradas, paralelas al eje del poste, sólidamente unidas a través de una matriz de resina epóxica. La fibra tiene aproximadamente 8 micrones de diámetro y actuando como un relleno del sistema. La matriz epóxica de BisGMA representas el 36\% del peso del poste. La interfase adherida entre estos 
materiales compatibles asegura que existirá una excelente adhesión entre todos los componentes. La estructura interna del poste absorbe el esfuerzo que es aplicado al complejo poste-corona y conduce la fuerza a través del eje longitudinal de la raíz remanente (18). Pero este tipo de distribución de las fibras paralelas al eje del poste de fibra de carbono tiene una resistencia a la fuerza transversal mucho menor comparado con la fuerza compresiva y aplicada en el eje de las fibras (19).

Los postes deben tener el mismo o similar módulo de elasticidad que la dentina radicular, para distribuir mejor las fuerzas aplicadas sobre la raíz y así poder tener una mayor resistencia a la fractura radicular. Este requerimiento parecen cumplirlo los espigos de fibra de carbono, cuyo módulo de elasticidad es de 21 Gpa (2,1 x $10 \mathrm{Mpa}$ ) y para la dentina radicular de $18 \mathrm{Gpa}(1,8$ x $10 \mathrm{Mpa})$. Si esto se compara con el módulo de elasticidad de la aleación de titanio que es $120 \mathrm{Gpa}$, la del acero inoxidable que es 200 Gpa; entonces hay menos posibilidad de fractura con los postes de fibra de carbono porque absorbe y disipa las diferentes fuerzas que se aplican en el diente restaurado $(11,18,19)$.

Sorensen y Engelman evaluaron la resistencia a la fractura radicular de espigos-muñones colados y postes Parapost ${ }^{\circledR}$. Encontraron que la máxima adaptación del espigo-muñón colado al conducto radicular aumentó la resistencia a la fractura radicular, además que los postes Parapost ${ }^{\circledR}$ de lados paralelos tenían una longitud de fractura radicular menor que los espigos-muñones colados (20). El sistema Parapost ${ }^{\circledR}$ es uno de los sistemas de postes prefabricados clínicamente más exitosos $(21,22)$. Otros estudios compararon la resistencia a la fractura entre espigo-muñón colado y postes de fibra de carbono, encontrando que los espigos-muñones colados fueron los que resistían más la fractura en comparación con los postes de fibra de carbono $(23,30)$.

Los dientes restaurados con poste prefabricados de fibra de carbono son los que presentan menor resistencia al stress frente a carga trasnversal en comparación a los otros postes (16). En estudios clínicos se observaron el comportamiento de dientes restaurados con postes de fibra encontrando un índice de fracasos de 2$3,2 \%$, siendo válida esta técnica como alternativa de uso clínico $(24,28)$. Otros autores concluyen que los dientes despulpados restaurados con materiales adhesivos no requieren la inserción adicional de postes $(25,26)$.

Isidor et al. compararon la fatiga de los postes Parapost ${ }^{\circledR}$, fibra de carbono y espigo-muñón colado sometidos a carga cíclica, encontrando que la mayor resistencia a la fractura la tuvieron las piezas restauradas con poste de fibra de carbono, seguidos de los postes Parapost ${ }^{\circledR} \mathrm{y}$ por ultimo de los espigos-muñones colados (27). Otros autores encontraron que los postes de fibra de carbono presentaron la menor resistencia y los postes de aleación de titanio la mayor resistencia frente a carga transversal $(29,43)$.

Los movimientos masticatorios son de carácter multidireccional y cíclico, los que básicamente según su dirección pueden estar dados por fuerzas transversales (se cree que estas destruyen la integridad de la unidad corona espigo-raíz), por fuerzas tensionales (en sentido vertical) y de torsión (giro sobre su eje) (13,31).

Existen varios tipos de tensión, una es la tensión compresiva (se produce cuando una carga tiende a comprimir un cuerpo) y la tensión por flexión (se presenta en fuerzas tangenciales). Las tensiones por comprensión y flexión que se generan sobre el diente están relacionadas con la angulación bucolingual que presentan dichas piezas $(32,33)$.

Si los postes debilitan al diente, los procedimientos restauradores que ayudan a preservar la vitalidad pulpar y eliminan la necesidad de postes, son deseables. La cantidad de estructura dentaria remante después de la terapia endodóntica y la técnica de preparación del poste son de importancia para el éxito del tratamiento (34).

Mientras menor es el grosor de la dentina residual en dientes anteriores superiores en sentido bucal es mayor la posibilidad de fractura radicular debido al tipo de fuerza a las que están sometidas. Los conductos con $1 \mathrm{~mm}$ de pared de dentina vestibular son más propensos a fracturarse bajo impactos horizontales de aquellos que tienen 2 ó 3 mm de pared dentinaria bucal (36).
No toda la estructura dentaria remanente contribuye por igual a dar resistencia a la pieza tratada endodonticamente. La mayor importancia radica en el milímetro más cervical de la corona clínica, cuyas paredes paralelas en complemento con la ulterior corona, proveen el llamado efecto zuncho o abrazadera, y no los biseles de la preparación para el muñón o para la corona $(20,39)$. Este efecto se consigue conservando al menos 1-2 mm de diente sano por encima de la terminación coronal. Se denomina efecto abrazadera a la circunscripción de una banda de metal alrededor de la superficie dentaría, cuya función es la de mejorar la integridad estructural de los dientes tratados endodonticamente, neutralizando el nivel de fuerzas funcionales, el efecto cuña de los postes cónicos y las fuerzas laterales durante la inserción (20,39,37,38).

La fractura radicular vertical es un problema clínico en piezas dentarias tratadas endodónticamente y se pueden producir durante la preparación biomecánica, en la preparación del espacio para el poste, por la excesiva presión hidráulica del cementado, por la excesiva fuerza en la condensación lateral y por el stress generado por el poste, lo cual puede condenar al diente a la exodoncia (6).

La localización de la fractura radicular se encuentra en el tercio cervical, medio y otras en el tercio apical de la raíz $(22,38,40)$.

Los picos de tensión-compresión ocurren en la dentina a nivel del tercio coronal de la raíz y los picos de stress por cizallamiento ocurren adyacentes al poste, a nivel de la mitad de la raíz en piezas uniradiculares. La magnitud del stress por cizallamiento esta influenciado por la dimensión y el largo del poste y este aumenta substancialmente a medida que el diámetro aumenta y largo decrece $(41,42)$.

El propósito del presente estudio fue determinar la resistencia a la fractura radicular de postes frente a carga estática transversal y de esta manera el clínico tenga la alternativa de elegir el mejor tipo de poste para evitar la perdida dentaria por fractura.

\section{Materiales y método}

El diseño del estudio fue de tipo ex 
perimental in vitro. El grupo experimental estuvo conformado por 30 premolares inferiores monorradiculares, las cuales fueron divididas en tres grupos de 10 piezas cada uno. Fueron tomados premolares inferiores monorradiculares cuya longitud total fue de $20 \mathrm{~mm}$, medidos desde la cúspide vestibular hasta el ápice de la raíz. La longitud de la raíz fue de $14 \mathrm{~mm}$ medidos desde la unión amelocementaria hasta el ápice de la raíz. Las 30 piezas dentarias premolares seleccionadas para el estudio fueron asignadas al azar en tres grupos de 10 piezas dentarias cada una y almacenadas en una solución de cloruro de sodio al $0,9 \%$ para mantener hidratada la dentina, durante todo el procedimiento (21).

Se confeccionó cubos de $32 \mathrm{~mm}$ de alto por $21 \mathrm{~mm}$ de largo por $21 \mathrm{~mm}$ de ancho, en cuyo centro se ubico las premolares con $1 \mathrm{~mm}$ de silicona de polivinilsiloxano Elite HD ${ }^{\circledR}$ (CZhermack Inc) en toda su periferia para imitar al ligamento periodontal, dejando expuesto $2 \mathrm{~mm}$ de la raíz medido desde el limite amelocementario, para simular el espacio biológico $(16,20)$.

Se tomaron radiografías del aspecto mesial de todas las piezas para la conductometría, conometría, obturación y adaptación de los espigos en el conducto, colocando el cono siempre a una misma distancia del objetivo. Se procedió a la preparación biomecánica con limas Flexofile ${ }^{\circledR}$ (C Maillefer Inc) hasta el número 45, lavando con hipoclorito de sodio al 2.5\% después de cada instrumentación. Se cambiaron las limas cada cinco tratamientos de conductos debido a la fatiga experimentada después del quinto tratamiento (21). Se obturaron los conductos con cemento tipo Grossman y conos de gutapercha mediante la técnica de condensación lateral. Se selló la entrada del conducto con un cemento temporal (Coltosol ${ }^{\circledR}$, (C)Coltène/Whaledent Inc) y se volvió a sumergir en la solución de cloruro de sodio al $0,9 \%$ por lo menos 24 horas antes de realizar la desobturación (21). Luego se desobturaron los conductos siguiendo las indicaciones de cada fabricante para el uso de cada kit, dejando ambos grupos en una longitud de desobturación de $11 \mathrm{~mm}$. Después se procedió a seccionar las coronas de las piezas dentales mediante un disco biactivo de diamante, dejando $2 \mathrm{~mm}$ de corona hacia oclusal medida desde la unión amelocementaria. Se lavó con suero fisiológico por 30 segundos utilizando una jeringa de 30cc, luego se seco con conos de papel (21).

El espigo-muñón colado fue hecho mediante la técnica directa, utilizando acrílico autopolimerizable Duralay ${ }^{\circledR}$ para confeccionar el patrón del espigo y del muñón. El patrón de acrílico se revistió con yeso de revestimiento de fosfato y la aleación utilizada fue no preciosa tipo III $\left(\mathrm{NPG}^{\circledR}\right)$. Los colados se retiraron del cilindro, se refinaron y se limpiaron con baño de arenado con óxido de aluminio de 50 ìm y presión de 2,8kg/cm² (16,17).

Luego se seleccionaron 10 postes de fibra de carbono de 1,4 mm de diámetro, de lados paralelos y lisos, marca CPost ${ }^{\circledR}$ (CBisco Inc), también se seleccionaron 10 postes de aleación de titanio N5 de lados paralelos y aserrados de 1,4 mm de diámetro, marca Parapost ${ }^{\circledR}$ (C) Coltène/Whaledent Inc). Los 10 postes Parapost ${ }^{\circledR}, 10$ postes C-Post ${ }^{\circledR}$ y los 10 espigos-muñónes colados fueron cementados utilizando el adhesivo All Bond $^{\circledR} 2$ (@Bisco, Inc) y la resina dual Duolink $^{\circledR}$ (@Bisco Inc). El conducto radicular fue grabado durante 15 segundos y luego lavado profundamente. El acondicionador All Bond $2^{\circledR}$ fue mezclado y aplicado sobre la dentina seca y grabada. Dos capas consecutivas fueron aplicadas al conducto radicular y a la estructura dentaria remanente, que fue secado durante 5 a 10 segundos para eliminar el solvente residual. El PREBOND RESIN del sistema All Bond $2^{\circledR}$ fue aplicado al conducto radicular y al resto de la estructura remanente, después se seco con jeringa de aire por cinco segundos. Fue aplicado dos capas del PRIMER B del sistema All Bond $2^{\circledR}$ al poste y se seco con aire. El cemento de curado dual Duolink ${ }^{\circledR}$ ( $($ Bisco Inc) fue mezclado y aplicado tanto al conducto radicular como al poste. El cemento resinoso fue llevado para todos los grupos al conducto radicular mediante el uso de un léntulo, después el poste fue colocado dentro del conducto radicular en la profundidad determinada, se polimerizó mediante luz halógena por 30 segundos. Para los grupos de postes C-Post ${ }^{\circledR}$ y Parapost ${ }^{\circledR}$ se colocó una matriz prefabricada de policarbonato. Las dimensiones de los muñones fueron de $3 \mathrm{~mm}$ de altura, $3 \mathrm{~mm}$ de largo mesiodistal y $5 \mathrm{~mm}$ de ancho vestibulolingual (21) y el muñón fue reconstruido con la resina Biscore ${ }^{\circledR}$ (CBisco Inc), fue polimerizado mediante luz halógena por 30 segundos. Los espigos fueron cementados a una presión constante de 2,72 kg por 5 minutos, se utilizó un paraleligrafo al cual se le confeccionó una cesta en la parte superior y se colocó un peso equivalente a 2,72 kg, esto permitió colocar el poste paralelo al eje axial de la pieza dentaria $(7,21)$.

Fue realizada la preparación dentaria dejando un hombro de $1 \mathrm{~mm}$ en toda la periferia y un bisel de 1mm de longitud y de 60 grados de inclinación. Se procedió a tomar las impresiones para la confección de las coronas mediante la técnica de la doble mezcla con polisiloxano Oranwash ${ }^{\circledR}$ y Zetaplus ${ }^{\circledR}$ (CZhermarck Inc.), mediante una cubeta hecha a medida.. Luego de media hora, las impresiones fueron vaciadas en yeso tipo IV Sylkirock ${ }^{\circledR}$ de (CWhip Mix Inc. Se hicieron coronas metálicas de una aleación no preciosa tipo III $\left(\mathrm{NPG}^{\circledR}\right)$ de un grosor de aproximadamente un milímetro de espesor y con inclinación cuspídea de 45 grados. Las coronas se arenaron en su parte interna con óxido de aluminio de 50 ìm y presión de 2,8 kg/cm² $(16,17)$. Fueron cementadas con ionómero-resina (Ketac ${ }^{\circledR}$ Cem, (C) 3 -ESPE AG) en un block de mezcla, a una presión constante de $2,72 \mathrm{~kg}$ por cinco minutos, finalmente fue protegida la interfase con el glaseador del Vitremer ${ }^{\circledR}$ (C3M-ESPEAG) de reconstrucción de muñones y se volvió a sumergir en la solución de cloruro de sodio al 0,9\% (21).

Fue utilizada la máquina de Hounsfild del Laboratorio de materiales de la Facultad de Ingeniería Mecánica de la Universidad Católica del Perú para ser sometidos a carga estática transversal. Antes de aplicar la carga, una persona ajena al estudio asignó aleatoriamente las letras A, B y C a cada grupo de estudio con sus respectivos números a cada muestras, así mismo la persona encargada de aplicar la fuerza con la maquina desconoció que tipo 
de poste estaba colocado en cada grupo experimental para conseguir que la prueba sea doble ciego. La carga estática transversal fue aplicada inclinando la pieza dentaria a 45 grados con respecto a su eje longitudinal, esto fue realizado para lograr que la carga incidiera perpendicular en la vertiente de la cúspide y que al momento de descomponer las fuerzas están fueran iguales, es decir que Fsen 45 = Fcos 45, así fue conseguido que ambas fuerzas sean iguales y que nin- guna predomine sobre la otra evitando agregar un sesgo a nuestra investigación. La fuerza resultante fue aplicada sobre la vertiente interna de la cúspide de soporte vestibular, a una distancia de $2 \mathrm{~mm}$ del centro de la fosa central de las coronas metálicas. Para tal fin fue utilizado un embolo de $5 \mathrm{~mm}$ de diámetro que fue graduado para una descarga con un incremento continuo de presión a una velocidad constante de $6.5 \mathrm{~mm} / \mathrm{min}$ hasta conseguir la fractura de la raíz (21).

Tabla 1. Análisis exploratorio de datos: media, desviación estandar, valor máximo, valor mínimo y coeficiente de variación de los grupos estudiados.

\begin{tabular}{lccc}
\hline & $\begin{array}{c}\text { Espigo-muñón } \\
\text { colado de titanio }\end{array}$ & $\begin{array}{l}\text { Poste de aleación Poste de fibra } \\
\text { de carbono }\end{array}$ \\
\hline Media & $221,0 \mathrm{~kg}-\mathrm{f}$ & $124,3 \mathrm{~kg}-\mathrm{f}$ & $60,8 \mathrm{~kg}-\mathrm{f}$ \\
Mediana & $227,5 \mathrm{~kg}-\mathrm{f}$ & $132,0 \mathrm{~kg}-\mathrm{f}$ & $61,0 \mathrm{~kg}-\mathrm{f}$ \\
D.S. & $31,9 \mathrm{~kg}-\mathrm{f}$ & $41,5 \mathrm{~kg}-\mathrm{f}$ & $18,9 \mathrm{~kg}-\mathrm{f}$ \\
Valor máximo & $260,0 \mathrm{~kg}-\mathrm{f}$ & $174,0 \mathrm{~kg}-\mathrm{f}$ & $108,0 \mathrm{~kg}-\mathrm{f}$ \\
Valor mínimo & $170,0 \mathrm{~kg}-\mathrm{f}$ & $66,0 \mathrm{~kg}-\mathrm{f}$ & $40,0 \mathrm{~kg}-\mathrm{f}$ \\
C.V. & $14,4 \%$ & $33,3 \%$ & $31,2 \%$ \\
\hline
\end{tabular}

Tabla 2. Comparación por pares de grupo con la prueba de Tukey.

\begin{tabular}{|c|c|c|}
\hline \multicolumn{3}{|c|}{ Par evaluado $\quad$ diferencia de medias (kg-f) Sig. } \\
\hline Espigo muñón colado- & & \\
\hline Poste de aleación de titanio & 96,7 & $<0,001^{*}$ \\
\hline Espigo muñón colado- & & \\
\hline Poste de fibra de carbono & 160,2 & $<0,001^{*}$ \\
\hline Poste de aleación de titanio- & & \\
\hline Poste de fibra de carbono & 63,5 & $<0,001^{*}$ \\
\hline
\end{tabular}

*Existe diferencia estadísticamente significativa $(\mathrm{p}<0.05)$ en la comparación por pares de grupos estudiados.

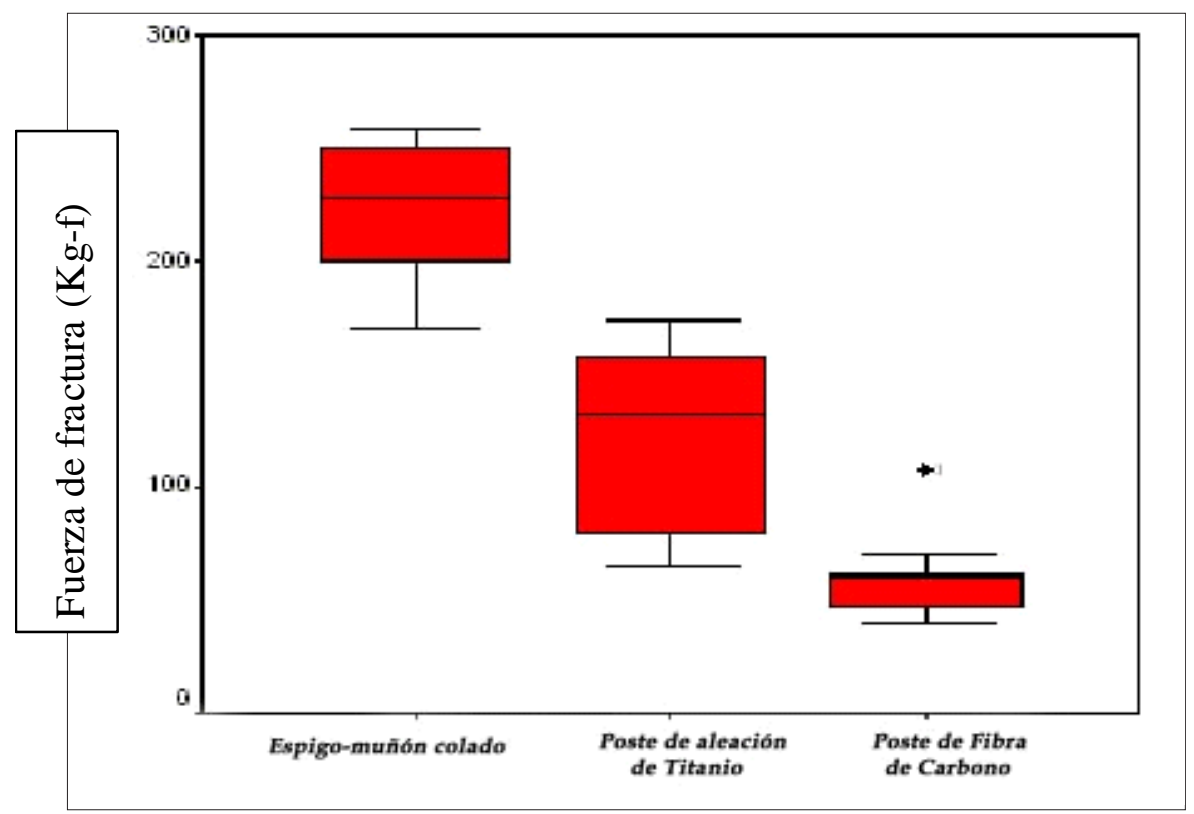

Figura 1. Fuerzas de fractura en las piezas dentarias reconstruidas con espigo-muñón colado, poste de fibra de carbono y de aleación de titanio.

Se recolectó la información en una ficha donde fue registrada la carga máxima hasta el momento en que fue producida la fractura de la raíz y su localización. Se realizó un análisis univariado de la resistencia a la fractura radicular para cada uno de los grupos haciendo un análisis descriptivo de los datos. Luego fue realizado un análisis bivariado para determinar la diferencia entre grupos; fue aplicada la prueba de análisis de varianza unifactorial. Para saber entre que grupos se produjo la diferencia, fueron comparados por pares mediante la prueba de Tukey.

\section{Resultados}

Las piezas dentarias restauradas con espigos-muñones colados fallaron a una fuerza de 221[IC 95\% (210,9; 231,1)] kg-f (Tabla 1).

Las piezas dentarias restauradas con postes de aleación de titanio fallaron a una fuerza de 124. 3 [IC 95\% (111,2; 137,4)] kg-f (Tabla 1).

Las piezas dentarias restauradas con postes de fibra de carbono fallaron a una fuerza de 60.8 [IC 95\% (54,9; 66,7)] kg-f (Tabla 1).

Según la prueba de Anova unifactorial, existen diferencias estadísticamente significativas entre los tres grupos comparados ( $\mathrm{p}<0,001)$. Mediante la prueba estadística para pares de los grupos conformados (prueba de Tukey) se vio que había diferencia estadísticamente significativa entre el grupo de espigo-muñón colado y poste de aleación de titanio $(\mathrm{p}<0,001)$, entre el grupo de espigo-muñón colado y poste de fibra de carbono $(\mathrm{p}<0,001)$ y entre el grupo de poste de aleación de titanio y poste de fibra de carbono $(\mathrm{p}<0,001)$ (Tabla 2) (Fig. 1).

En los tres grupos la localización de la fractura fue en el tercio apical de la raíz.

\section{Discusión}

Numerosos estudios defienden el uso de postes para la retención de muñones protésicos, pero no apoyan el uso de postes para fortalecer las piezas dentarias $(26,27,35)$.

Las investigaciones sobre postes han sido orientadas a encontrar materiales que tengan un módulo de elasticidad muy semejante a la dentina, para 
así poder preservar la dentina radicular, no someter a fuerzas excesivas a la raíz, resistentes a la corrosión y compatibles con los materiales restauradores. Entre estos materiales se encuentra los espigos prefabricados de fibra de carbono y de titanio $(1,6,10,14,24,27,28)$.

En nuestro estudio se encontró que las piezas dentarias restauradas con espigo-muñón colado resistió la mayor fuerza a carga estática transversal (Fig. 1). Estos resultados son similares a los encontrados por Sirimai et al. (22) quienes compararon la resistencia a la fractura radicular sometidos a carga estática transversal de seis sistemas de postes, en donde el grupo reconstruido con espigo-muñón colado resulto con mayor resistencia a la carga estática transversal en comparación con los otros sistemas. También en el trabajo de ZhiYue et al. (29), en donde compararon la resistencia a la fractura radicular con diferentes tipos de postes y su relación con el efecto zuncho, encontraron que el grupo restaurado con espigo-muñón colado con efecto zuncho fue el que tuvo mayor resistencia frente a carga estática transversal, seguido del grupo restaurado con poste prefabricado con efecto zuncho y por ultimo el grupo restaurado con espigo-muñón colado sin efecto zuncho.

Esto nos sugiere que es importante la adaptación intima del espigo-muñón colado dentro del conducto además de dejar los dos milímetros de dentina por encima del hombro para darle el efecto zuncho, lo cual parece contribuir en distribuir correctamente la fuerzas a través del eje dentario $(15,29)$. Esto tiene sustento en los resultados de Sorensen y Engelman (20,39), donde ellos sugirieron mantener $2 \mathrm{~mm}$ de estructura dentaría más arriba del hombro y una adaptación intima del espigo-muñón colado al conducto, por que demostraron que esta preservación aumenta la resistencia a la fractura radicular.

Las piezas dentarias restauradas con postes prefabricados de aleación de titanio mostró una mayor resistencia frente a carga estática transversal con respecto a las piezas dentarias restauradas con espigo de fibra de carbono (Tabla 2, Fig. 1), pero una menor resistencia frente a carga estática transversal en comparación a las piezas restauradas con espigo-muñón colado. Estos resultados son similares a los encontrados por Sidoli et al. (16), quienes encontraron que el poste Parapost ${ }^{\circledR}$ sometido a carga estática transversal tiene una resistencia mayor frente a carga estática transversal que los postes de fibra de carbono, siendo el espigo-muñón colado el que tuvo mayor resistencia frente a carga transversal. Esto puede deberse a que el poste de titanio por su modulo de elasticidad, su forma cilíndrica y de lados paralelos distribuye mejor las cargas $(1,10,14,18,27,28)$.

Las piezas dentarias restauradas con poste de fibra de carbono fue el que tuvo una resistencia baja frente a carga estática transversal en comparación a los otros grupos (tabla 2 y figura 1). Estos resultados son similares a los encontrados por Purton et al. (43) quienes encontraron que el poste de fibra de carbono tuvo niveles de resistencia mucho menor en comparación con los postes Parapost ${ }^{\circledR}$. También en los estudios de Ludi et al. (23) y Martínez-Insua et al. (30) el grupo restaurado con poste de fibra de carbono resiste menos frente a carga estática transversal que el grupo restaurado con espigo-muñón colado. Esto puede deberse a que la resistencia de la fibra de carbono se encuentra en valores bajos frente a carga estática transversal según lo indica el fabricante y corroborado por los estudios de Duret et al. (19) y Purton y Love (43). Esto puede explicar los valores bajos encontrados en el grupo de espigo de fibra de carbono y de aleación de titanio $(7,34)$.

Si comparamos nuestros resultados de resistencia a la fractura radicular frente a carga estática transversal con otros estudios en donde aplicaron carga cíclica, observaremos que los que mejor se comportan son los postes de fibra de carbono, debido a que la carga cíclica evalúa la fatiga del material para después ocurrir la fractura, en cambio la carga estática evalúa la resistencia del material frente a una fuerza aplicada hasta ocurrir la fractura (27).

Si estos resultados los comparamos con los estudios de Fredriksson et al. (28) y Ferrari y Scotti (34), es válido el uso clínico de los postes de fibra de carbono por tener un índice de fracasos clínicos muy bajos. Esto se puede deber a que los postes de fibra de carbono tienen una mayor resistencia a la fatiga, como lo de- mostraron los estudio de Oliveira (25) e Isidor et al. (27), además que la carga máxima de un individuo a nivel de premolares es de 46 kg-f y en nuestro estudio los postes de fibra de carbono fallaron a los 108 kg-f como valor máximo y 48 kg-f como valor mínimo (5).

El modo de falla de los postes puede producirse por fractura o curvatura de los postes, pérdida de retención, fractura del muñón o fractura radicular $(6,27,39)$. En nuestro estudio los tres grupos presentaron la fractura en el tercio apical de la raíz, esto se pudo deber a que la transmisión de las fuerzas transversales puede dirigir el esfuerzo hacia áreas específicas de la raíz, por presentar el fulcrum a ese nivel debido a carga transversal (35). Los espigos-muñones colados presentaron una mayor perdida de dentina radicular frente a la fractura que los postes de aleación de titanio y de fibra de carbono. Estos resultados también fueron encontrados en los estudios realizado por Sirimai et al. (22), Assif et al. (38) y Gluskin et al. (40).

\section{Referencias bibliográficas}

1. Urquizo G. Rehabilitación del diente despulpado.Odontologia Uruguaya 1995; 63:29-32.

2. Angulo MM, Lara RH. Sistema perno muñón vaciado metálico. Rev Dent Chile 1996; 87:47-55.

3. Dell'Acqua H, Parodi G Mauttoni N. Pernos radiculares colados y de stock. Estudio comparativo. Rev Odonto Uru 1994; 42:4-10.

4. Standlee JP, Caputo AA. Endodontic dowel retention with resinous cements. J Prosthet Dent 1992; 68:9137.

5. Kahn FF, Rosenberg P,Schulman A, Pines M. Comparison of fatigue for three prefabricated threaded postlsystem. J Prosthet Dent 1996; 75:148-53.

6. Morgano SM, Milot P. Clinical sucess of cast metal post and cores. J Prosthet Dent 1996; 69:11-6.

7. Dávila E. Estudio comparativo de la retención de espigos colados con aleación $\mathrm{Cu}-\mathrm{Al}$ vs. un tipo de espigo prefabricado cónico con estrías. [Tesis para optar por el grado de Cirujano-Dentista]. Lima: Univ. Peruana Cayetano Heredia, 1990.

8. OBrien R. Materiales Dentales y su 
selección.3ra Ed. Madrid: Panamericana, 1980.

9. Phillips W. La ciencia de los materiales dentales de Skiner. 4ta Ed. México D. F: Interamericana.-Mc Graw Hill, 1993.

10. Martínez RA. Comparación de la resistencia a la fractura de dientes con tratamiento de conductos conservador, restaurador con amalgama dental, ionómero de vitreo y resina compuesta [Tesis para optar el título de Cirujano-Dentista]. Lima: Univ. Peruana Cayetano Heredia, 1994.

11. Stegarou R, Yamada H, Kusakari H, Miyakawa O. Retention and failure modes after cyclic loading in two post and core systems. J Prosthet Dent 1996; 75:120-5.

12. Ingle J, Traintor J. Endodoncia: 3er Ed. México D.F: Interamericana, 1987.

13. Cohen BI, Pagnillo MK, Newman I,Musikant BL, Deutsch A. Retention of three endodontic post cemented with five dental cements. J Prosthet Dent 1998; 79:520-5.

14. Angulo MM, Irribarra RS. Sistema perno muñón mixto de resina compuesta y metal: Revisión de la técnica de una decada. Rev Dent Chile 1996, 87:47-55.

15. Henostroza GH. Adhesión en Odontología Restauradora. 1ra Ed. Sao Paulo :Maio, 2003.

16. Sidoli G, King P, Setchell D. An in vitro evaluation of carbon fiberbased post and core system. J Prosthet Dent 1997; 78:5-9.

17. Morfis A, Athens G. Vertical root fracture. Oral Surg Oral Med Oral Pathol. 1990; 69:631-5.

18. Freedman G. Los postes de fibra de carbono : Rehabilitación postendodóntica adhesiva. J Clínica en Odontología 1997; 2:19-26.

19. Duret B, Reynaud M, Duret F. Un nouveau concept de reconstitution corono-radiculare : Le Composipost (1). Le chirurgien-dentiste de France 1990; $540: 131-41$.

20. Sorensen JA. Engelman MJ. Effect of post adaption of fracture resístanse of endodontocally treated teeth. J Prosthetic Dent 1990; 64:190-99.

21. Torbjorner A, Karlsson S, Odman P.
Survical rate and failure characteristics for to post designs. J Prosthet Dent 1995; 73:439-44.

22. Sirimai S, Riis DN, Morgano SM.An in vitro study of the fracture resistance and the incidence of vertical root fracture of pulpless teeth restored with six post-core systems. J Prosthet Dent 1999; 81:262-9.

23. Ludi V, Gonzáles J, Varela O, Rivas P, Paz F, Gude F. Estudio comparativo de la resistencia a las fuerzas de cizalla entre pernos muñones colados y prefabricados de fibra de carbono. Quintessence 1998; 11: 652- 56.

24. Ferrari M, Vichi A, Mannocci F, Manson P. Restrospective study of the clinical performance of fiber posts. Amer J Dent 2000; 13: 9-13.

25. Oliveira UM. Avaliaciòn da resistencia de la fractura de dientes endodonticamente tratados restaurados con resina compusta y pino de fibra de carbono, estudio in vitro [Tesis de Maestría]. Brasil: Univ. Luterana de Brasil, 2003.

26. Heydecke G, Burtz F, Hussein A, Strub J. Fracture strength after dynamic loading of endodonticaly treated teeth restored with different post and core systems. J Prosthet Dent 2002; 87:438-45.

27. Isidor F, Odman P, Brodum K. Intermittent loading of teeth restored using prefabricated carbon fiber. Int J Prosthodont 1996; 9 :131-36.

28. Fredriksson M, Astback J, Pamenius M, Arvidson K. A retrospective study of 236 patients with teeth restored by carbon fiber-reinforced epoxy resin posts. J Prosthet Dent 1998; 80: 151-57.

29. Zhi-Yue L, Yu-Xing Z. Effects of post-core design and ferrule on fracture resistance of endodonticatically treated maxillary central incisors. J Prosthet Dent 2003; 89: 368-73.

30. Martinez-Insua A. Da Silva L, Rilo B, Santana U. Comparison of the fracture resistances of pulpless teeth restored with a cast post and core or carbon-fiber post with a composite core. J Prosthet Dent 1998; 80: 527-32.

31. Kurer $H$. The classification of singlerooted, pulpless teeth. Quintessence Int 1991; 22: 939-43.
32. Canut, J. Ortodoncia Clínica.3ra Ed. Madrid: Salvat,1992.

33. Ho M, Lee S. Three dimensional finite element analysis of the effects of post on stress distribution in dentin. J Prosthet Dent 1994; 72:367-72.

34. Ferrari M, Scotti R. Pinos de Fibra: Consideracoes teóricas e aplicacoes Clínicas.1ra Ed. Sao Paulo: Artes Medicas, 2003.

35. Castilla M. Resistencia a la fractura en piezas dentarias con tratamiento de conductos restaurados con espigos. Estudio comparativo experimental [Tesis para optar el título de Cirujano-Dentista].Lima: Univ. Peruana Cayetano Heredia, 1997.

36. Tjan AH, Nemetz H. Effect of eugenol-containning endodontic sealer on retention of prefabricated post luted with an adhesive composite resin cemet. Quintessence Int 1992; 23: 839-44.

37. Henostroza G. Criterios actuales para restauraciones de piezas depulpadas. Publicación de la Fundación de Investigación y servicio Estomatológico "Oswaldo Chavez Jaramillo" Ecuador 1994; 1: 27-32.

38. Assif D, Bitenski A, Pilo R, Oren E .Effect of post design on resistance to fracture of endodontically treated teeth with complete crowns. J Prosthet Dent 1993; 69:36-40.

39. Sorensen JA, Engelman MJ. Ferrule desing and fracture resistence of endodontically treated teeth. J Prosthet Dent 1990; 63: 25-36.

40. Gluskin A, Radke R, Frost S, Watanabe L. The mandibular Incisor: Rethinking guidelines for post and core design. J Endodontics 1995; 21: 33-7.

41. Holmes D, Díaz-Arnold A,Leary J. Influence of post dimension on stress distribution in dentin. J Prosthet Dent 1996; 75: 140-7.

42. Mori M, Ueti M, Matson E, Saito T. Finite element análisis of internal stress distribution, Ander axial force, in sound tooth, and in tooth restored with metalloceramic crown and cast metal post and core. Rev Odontol Univ Sao Paulo 1997; 11: 99-107.

43. Purton DG, Love RA. Rigidity and retention of carbon fibre versus stainless steel root canal posts. Int J Endodontics1996; 29: 262-5. 